\title{
Caracterización socioeconómica de fincas cafeteras del departamento de Nariño, Colombia
}

\section{Socioeconomic characterization of coffee farms of the department of Nariño, Colombia}

\author{
Hernando Criollo Escobar; Deysy Benavides Arteaga²; Johanna Muñoz Belalcázar; Tulio César Lagos Burbano ${ }^{4}$
}

\begin{abstract}
IIIng. Agrónomo Ph.D. Universidad de Nariño, sede VIPRI, Facultad de Ciencias Agrícolas, Grupo de Investigación en Producción de Frutales Andinos GPFA. San Juan de Pasto - Nariño, Colombia; e-mail: hcriollo@udenar.edu.co; Dhttps://orcid.org/0000-0002-8690-4486

${ }^{2}$ Ing. Agroforestal. Universidad de Nariño, sede VIPRI, Facultad de Ciencias Agrícolas, Grupo de Investigación en Producción de Frutales Andinos GPFA. San Juan de Pasto - Nariño, Colombia; e-mail: dvbenavides@gmail.com; Dhttps://orcid.org/0000-0002-3549-1343
\end{abstract}

${ }^{3}$ Ing. Agrónomo, M.Sc. Universidad de Nariño, sede VIPRI, Facultad de Ciencias Agrícolas, Grupo de Investigación en Producción de Frutales Andinos GPFA. San Juan de Pasto - Nariño, Colombia; e-mail: mjohannaalixa@gmail.com; Dhttps://orcid.org/0000-0003-4466-192X

${ }^{4}$ Ing. Agrónomo. Ph.D. Universidad de Nariño, sede VIPRI, Facultad de Ciencias Agrícolas, Grupo de Investigación en Producción de Frutales Andinos GPFA. San Juan de Pasto - Nariño, Colombia; e-mail: tclagosb@udenar.edu.co; Dhttps://orcid.org/0000-0001-9222-4674

Cómo citar: Criollo Escobar, H.; Benavides Arteaga, D.; Muñoz Belalcázar, J.; Lagos Burbano, T.C. 2019. Caracterización socioeconómica de fincas cafeteras del departamento de Nariño, Colombia Rev. U.D.C.A Act. \& Div. Cient. 22(2):e1397. http://doi.org/10.31910/rudca. v22.n2.2019.1397

Artículo de acceso abierto publicado por Revista U.D.C.A Actualidad \& Divulgación Científica, bajo una licencia Creative Commons CC BY-NC 4.0

Recibido: Junio 25 de 2018

Aceptado: Noviembre 11 de 2019

Editado por: Ingeborg Zenner de Polanía

\section{RESUMEN}

La adopción de tecnologías generadas para el cultivo del café depende, en gran medida de factores, como la disponibilidad de recursos y el nivel de escolaridad de los productores, lo cual, determina la importancia de analizar las condiciones socioeconómicas, en la estructuración de los sistemas productivos de café. El objetivo de esta investigación fue analizar las principales características sociales y económicas de los cafeteros en los ecotopos 220A y 221A, departamento de Nariño. Con base en un marco muestral de 16.767 predios cafeteros, se seleccionaron aleatoriamente 159 productores (86, del ecotopo 220A y 73, del 221A), con el objeto de aplicar el formulario de encuesta. En el análisis estadístico, 58 variables categóricas fueron sometidas a un análisis multivariado, mediante el método de correspondencias múltiples y análisis de clasificación jerárquica. El ecotopo 220A, se caracterizó por tener áreas de café entre 1 y 3 hectáreas, viviendas con techos de eternit y zinc, pisos de cemento, energía eléctrica, acueducto y unidad sanitaria. El rendimiento está entre 1.001 y $2.000 \mathrm{~kg} \cdot \mathrm{ha}^{-1}$, costos de producción menores a COP1.500.000 (USD444,83). Los caficultores asisten a jornadas de capacitación y su núcleo familiar está compuesto por 4-7 personas. En el ecotopo 221A predominan las casas con techo de teja, pisos en cemento, energía eléctrica, acueducto y unidad sanitaria; las aguas servidas se disponen en pozos sépticos. No se aplican las buenas prácticas agrícolas (BPA), los rendimientos son menores a $1.500 \mathrm{~kg} \cdot \mathrm{ha}^{-1}$ cps (café pergamino seco) y los costos de producción son inferiores a COP1.500.000 ha $\mathrm{haño}^{-1}$ (USD444,83).

Palabras clave: Coffea arabica L.; ecotopos cafeteros; análisis multivariado; zona agroecológica. 


\section{ABSTRACT}

The adoption of technologies generated for the coffee crop, depends to a great degree on factors such as the availability of resources and the producer's scholarship which determines the importance of socioeconomic conditions in the coffee productive system structure. The objective of this investigation was to analyze the main coffee grower's social and economic characteristics in the $220 \mathrm{~A}$ and 221A Nariño Department ecotopes. 159 producers (86 from the ecotype 220A and 73 from the ecotope 221A), were selected randomly based on a sampling frame of 16.767 coffee farms, with the purpose of applying the survey questionnaire. In the statistical analysis, 58 categorical variables were used, they were subjected to a multivariate analysis using the multiple correspondence method and hierarchical classification analysis. The 220A ecotope was characterized by having coffee areas between 1 and 3 hectares, houses with eternit and zinc roofs, cement floors, electric power, aqueduct and sanitary unit. The performance is between 1.001 and 2.000kg.ha ${ }^{1}$, the cost of production is less than COP1.500,000 (USD44,83); the coffee growers attend training sessions and their family nucleus is made up of 4 - 7 people. In the ecotope $221 \mathrm{~A}$ the houses with tile roof, cement floors, electricity, aqueduct and sanitary unit predominate; wastewater is disposed of in septic tanks. Good agricultural practices (GAP) aren't used, the yields are less than $1.500 \mathrm{~kg} \cdot \mathrm{ha}^{-1}$ (dried parchment coffee) and the cost of production is less than $\$ 1.500 .000 \mathrm{ha}^{-1}$. year

Keywords: Coffea arabica L.; ecotypes; multivariate análisis; agroecological zone.

\section{INTRODUCCIÓN}

Según la Organización Internacional del Café (OIC, 2016), para el periodo 2015-2016, la producción mundial de café pergamino seco (cps) fue de 8,88 millones de toneladas, con un aumento del 0,9\% respecto al periodo 2014-2015; esta producción, ubica, en primer lugar, a Brasil, con 2,3 millones de toneladas, seguido de Vietnam, con 1,7 millones de toneladas y Colombia, con 840 mil toneladas. El sector cafetero ocupa un renglón importante en la economía mundial (Hernández, 2009), ya que se produce en más de 80 países de Latinoamérica, África y Asia (Panhuysen \& Pierrot, 2014). Desde mediados del siglo XIX, el sector cafetero colombiano, se ha convertido en uno de los pilares de la economía del país, ocupando los primeros lugares entre los productos agropecuarios destinados a la exportación (Piñeros, 2016).

El café es uno de los productos más importantes de la agricultura, debido a la demanda que existe por parte de los consumidores. El cultivo, se caracteriza por requerir mucha mano de obra familiar, con predominio de mujeres y por cultivarse en pequeña escala (Panhuysen \& Pierrot, 2014); contribuye a la subsistencia de más de 20 millones de familias campesinas e involucra a más de 100 millones de personas en su producción, pos-cosecha y comercialización (McNougher, 2013).

En Colombia, para el 2016, el área establecida con café era de 777.704ha cultivadas (Agronet, 2018), generando cerca de 785 mil empleos directos en el sector rural, correspondiente al 26\% de los empleos generados por las actividades agrícolas (FNC, 2014b). Para este mismo periodo, Nariño contaba con 32.750ha de café en producción, que representan, aproximadamente, el 4,21\% del área cafetera nacional, con un rendimiento de $1,09 \mathrm{t}^{-h \mathrm{a}^{-1}}$, superior al promedio nacional $\left(1,06 \mathrm{t} \cdot \mathrm{ha}^{-1}\right)$.

Durante los últimos años, las políticas cafeteras del país han promovido el consumo, la consecución de nuevos mercados y el fortalecimiento de la educación rural, con miras al continuo crecimiento del producto en los diferentes mercados y con perspectivas de mejorar la calidad de vida de los caficultores (FNC, 2014b).

De igual forma, la Federación Nacional de Cafeteros, en alianza con sus clientes y financiadores, como Nestlé, Nespresso y Cenicafé, ha desarrollado proyectos sociales, enfocados a promover la sostenibilidad del ingreso de las familias cafeteras, mediante programas y proyectos de inversión, en competitividad, en infraestructura comunitaria y domiciliaria, en educación, en capacitación, en protección del medio ambiente, en protección social, en fortalecimiento gremial y en conectividad (FNC, 2014b).

En Nariño, esta actividad genera, anualmente, 27.000 empleos directos (FNC, 2014a); el 40\% del suelo de la zona cafetera nariñense, se encuentra cultivado con café y el 60\% restante cuenta con cultivos de caña panelera, maíz, fríjol, plátano, reservas forestales y pastos no tecnificados (CCN, 2014). La distribución de la tierra es minifundista; el 86,5\% de los predios rurales tienen menos de 10 has (CONPES, 2014); en cuanto a la producción cafetera de Nariño, se da en predios que, en un $96 \%$, son menores de una hectárea y solo una porción de la misma es utilizada en el cultivo de café (Burgos, 2015). La distribución de la tierra es básicamente minifundista y la tenencia de la tierra es del $80 \%$, propietarios; el 18\%, aparceros y el 2\%, arrendatarios (Viloria, 2007).

Existen cultivos de café en 41 de los 65 municipios del departamento, donde hay, aproximadamente, 54.772 fincas, con un estimado de 39.423 productores, que representan el $7,1 \%$ de los cafeteros del país (CCN, 2014; FNC, 2016).

El manejo del cultivo en Nariño, se realiza de forma tradicional, estrechamente relacionado con la cultura y la economía familiar. En la actualidad, tras la ejecución de programas fomentados por la Federación Nacional de Cafeteros y el Gobierno Nacional, alianzas con entidades privadas, subsidios a productores pobres y créditos cafeteros, se ha tecnificado un alto porcentaje de las pequeñas fincas; sin embargo, el nivel de tecnificación no es elevado, pues, en muchos casos, solo es soportado por asesorías técnicas y sistemas de riego, medianamente tecnificados (Burgos, 2015); la asistencia técnica es limitada, se usan técnicas tradicionales de beneficio y de poscosecha y el apoyo en infraestructura, en equipos y en maquinaria para el procesamiento del grano, es limitado (Rodríguez-Camayo et al. 2015; Martínez, 2004). A pesar de las condiciones precarias y las dificultades técnicas y de comercialización de los caficultores nariñenses, el departamento de Nariño cuenta con potencial en 
la producción de café de excelente calidad tipo Gourmet, con características diferenciadoras en sabor y en aroma (Burgos, 2015), que le han permitido posicionarse en los diferentes mercados internacionales. Con la catalogación del café de Matituy, como el mejor café del mundo, en los años 50, se inició un trabajo arduo para mantener las características que le otorgan una calidad especial (FNC, 2010), logrando diferentes reconocimientos, a nivel mundial y una aceptable estabilidad socioeconómica de las familias cafeteras. La connotación de café especial es una alternativa de los productores del sur, para consolidarse en los mercados internacionales.

La caficultura es el proyecto de vida de muchos productores, que confían en que este commodity (materia prima o bien transable en el mercado de valores) continúe, como uno de los más importantes para muchas economías emergentes (Paiva, 2000; Hatanaka et al. 2005; Maurice \& Davis, 2011). A pesar de las dificultades, muchas familias dependen del cultivo del café como medio de sustento y han desarrollado toda una cultura del café, de donde provienen varias de sus tradiciones y prácticas, ligadas a la cotidianidad de su cultivo, el procesamiento y la distribución (Silva \& Trejos, 2016).

La generación de planes de mejoramiento orientados al bienestar de los productores del sector cafetero, requiere del conocimiento real de la situación socioeconómica de estos agricultores, razón por la cual, se realizó este estudio, a partir de la pregunta ¿Cuáles son y cómo se relacionan las variables socioeconómicas que definen al sector cafetero del departamento de Nariño?

\section{MATERIALES Y MÉTODOS}

La investigación, se realizó en el 2015, en los ecotopos cafeteros 220A (Latitud Norte: $1^{\circ} 21^{\prime}$ - 142’) (La Unión y Buesaco) y 221A (Latitud Norte $1^{\circ} 05^{\prime}-1^{\circ} 36^{\prime}$ ) (La Florida, Sandoná y Consacá), localizados en el departamento de Nariño. Inicialmente, se revisaron estadísticas de la Federación Nacional de Cafeteros de la región, que permitieron establecer un marco muestral de 16.767 unidades de productivas cafeteras (UPC) y un tamaño de muestra de 159 UPC, mediante la utilización de la fórmula propuesta por Scheaffer et al. (2007). Para la distribución de las 159 encuestas, se tuvo en cuenta el porcentaje de participación de las UPC en los dos ecotopos; en el ecotopo $220 \mathrm{~A}$, equivalente al 54,3\% del total de cultivadores de café en los municipios citados (86 encuestas) y en el ecotopo 221A, correspondiente al 45,7\% (73 encuestas).

Para la recolección de la información, se utilizó un formato de encuesta, diligenciado mediante entrevista personal y observación directa de las UPC. El formulario de encuesta contempló 58 variables, las cuales, eran de tipo económico (24), social (29) y tecnológico (5). Se definieron con base en el sistema productivo de café, orientadas a la identificación y valoración de aspectos relacionados con: tenencia de tierra, componentes de la finca, productividad, manejo técnico del cultivo, proceso de cosecha y poscosecha, estimativo de costos de producción, comercialización del producto, recurso humano, gestión agroempresarial, integración de actores de la cadena, instituciones de apoyo, organización social, grupos etarios, educación, vivienda, infraestructura y conocimiento de políticas agropecuarias, cuyas respuestas fueron estructuradas de forma categórica, para la aplicación de un análisis de correspondencias múltiples (ACM) y análisis de clasificación, con base en las distancias de Ward, utilizando el software SPAD 5.6 (Ríos et al. 2004; Chávez et al. 2010).

\section{RESULTADOS Y DISCUSIÓN}

Ecotopo 220A. El análisis descriptivo de las variables cualitativas para el ecotopo cafetero $220 \mathrm{~A}$ mostró que un 74,4\% de las fincas tienen menos de 3has cultivadas con café, confirmándose el carácter minifundista de los cafeteros, expresado por Becerra (2010) y Dussán et al. (2006), que afirman que los predios con áreas menores a 5 has en café representan el $95,2 \%$ de las fincas del país. El 50\% de las viviendas tienen techos de eternit y zinc, predominando los pisos de cemento (62,8\%); el 83,7\% de las viviendas cuentan con servicios públicos de energía eléctrica y acueducto; el 84,9\% tienen unidad sanitaria y un 57\%, cocinan con gas. En contraste, Aristizabal \& Duque (2008) reportan que el 100\% de los productores de la zona central cafetera en Colombia cuentan con energía eléctrica; un $64 \%$, con acueducto; un $67 \%$, con línea telefónica y la fuente de combustible más usada para cocinar, es gas.

En cuanto a la producción, el 72,1\% de las UPC producen entre 1.001 - 2.000kg.ha ${ }^{-1}$ y el 38,4\% (se podría poner medias y la desviación estándar, por ejemplo, $1.000 \pm 300 \mathrm{kgha}-1$ ) de los productores tienen costos inferiores a COP1.500.000ha ${ }^{-1}$ (USD544,83); el 62,8\% de los caficultores asisten a capacitaciones, realizadas por instituciones de economía mixta (61,6\%). Un 52,3\% de las viviendas están habitadas por familias compuestas entre 4 y 7 personas y un $44,2 \%$, poseen solo educación primaria.

El análisis del histograma de valores propios (Tabla 1) permitió seleccionar los primeros tres factores que explican, en conjunto, el 34,9\% de la variabilidad, debida a las variables cualitativas. El análisis de correlación variable-factor (Tabla 2) permitió establecer que las variables que más contribuyeron a la conformación del factor 1 , fueron: tipo de techo, tipo de piso, combustible para cocinar, servicios domésticos y sanitarios y número de personas que viven en la finca. Estos resultados demuestran que las variables de tipo social son las más importantes en la conformación de este componente. Las principales variables que aportaron a la conformación del factor dos, fueron: extensión de la finca, área cultivada en café, entidades que realizan las capacitaciones, pertenencia a organizaciones de cafeteros, participación en capacitaciones, lo cual, permite catalogar a este factor como cultural.

Las variables que hicieron el mayor aporte al factor tres, fueron: extensión de la finca, área cultivada con café, asistencia técnica, capacitaciones y costos de insumos ha.año ${ }^{-1}$, considerando este factor como económico. La ubicación de las variables de tipo social en el componente 1 destaca la importancia de lo social como un indicador que explica, en gran medida, el estado actual de los cafeteros de Nariño; asimismo, determina la importancia de priorizar y de valorar el componente social, en los diferentes proyectos planificados para el sector cafetero en el departamento, ya que, según 
Tabla 1. Representación porcentual de la diversificación observada en las variables cualitativas relacionadas con las condiciones socioeconómicas de los caficultores del ecotopo 220A.

\begin{tabular}{|c|c|c|c|l|}
\hline Factor & $\begin{array}{c}\text { Valor } \\
\text { Propio }\end{array}$ & Porcentaje & $\begin{array}{c}\text { Porcentaje acumu- } \\
\text { lado }\end{array}$ & \multicolumn{1}{|c|}{ Histograma } \\
\hline 1 & 0,3127 & 15,63 & 15,63 & $* * * * * * * * * * * * * * * *$ \\
\hline 2 & 0,2544 & 12,72 & 28,35 & ************ \\
\hline 3 & 0,1309 & 6,55 & 34,90 & $* * * * * *$ \\
\hline
\end{tabular}

Tabla 2. Correlación entre las variables más aportantes y cada uno de los factores principales en el ecotopo 220A.

\begin{tabular}{|l|c|c|c|}
\hline \multirow{2}{*}{\multicolumn{1}{|c|}{ Variables }} & \multicolumn{3}{c|}{ Factores } \\
\cline { 2 - 4 } & $\mathbf{1}$ & $\mathbf{2}$ & $\mathbf{3}$ \\
\hline Extensión de la finca (ha) & 0,1 & 9,7 & 13,2 \\
\hline Área sembrada en café (ha) & 1,1 & 10,2 & 14 \\
\hline Tipo de techo & 10 & 1,8 & 5,6 \\
\hline Tipo de piso & 12,3 & 3 & 3,7 \\
\hline Combustible para cocinar & 15,8 & 2,4 & 0,6 \\
\hline Servicios domésticos & 12,5 & 0,5 & 0 \\
\hline Servicio sanitario & 11,8 & 0 & 3,4 \\
\hline Recibe algún tipo de asistencia técnica & 0,3 & 10,8 & 10,7 \\
\hline Pertenece a alguna Cooperativa, Asociación, & 0 & 10 & 4,4 \\
\hline Fundación o Certificadora de calidad de café & & & 8,7 \\
\hline Asiste a capacitaciones sobre el cultivo & 0,7 & 11 & 9,8 \\
\hline Que instituciones realizan las capacitaciones & 1,8 & 12,2 & 3,6 \\
\hline Número de personas que viven en la finca & 14,9 & 1,3 & \\
\hline
\end{tabular}

Bravo-Monroy et al. (2016), la identidad social de los productores, la gestión de las instituciones y las relaciones sociales, son determinantes en el proceso de decisiones. El análisis de clasificación del ecotopo cafetero 220A del departamento de Nariño permitió conformar tres grupos; los individuos agrupados en cada clase, se observan en la figura 1. El Grupo 1 conformado por 39 productores, quienes representan el 45,34\% del total, se caracterizó porque el 82,05\% utilizan gas como combustible; el $100 \%$ de los productores reciben capacitaciones de empresas de economía mixta, donde el 66,97\% la recibe de la Federación Nacional de Cafeteros; el 97,44\% asisten a capacitaciones; el 74,36\% tienen alianzas comerciales y el 76,92\% pertenecen a organizaciones cafeteras; el $71,79 \%$ de los productores conocen y aplican BPA.

Según la FNC (2013), la articulación de pequeños productores aporta al crecimiento y desarrollo de la agricultura, contribuyendo a la creación de condiciones de progreso, de equidad y de vida digna de los habitantes rurales, reduciendo la pobreza y la inequidad en el campo. Asimismo, la creación de instituciones, como la FNC y de un conjunto de organizaciones prestadoras de servicios a la industria cafetera, como la empresa Alianza Café Nariño SAS, conformada por 11 asociaciones, con 2.500 caficultores, pertenecientes a 14 municipios, donde existen microprocesadores de café, han contribuido, considerablemente, al desarrollo del sector cafetero (De La Rosa, 2011).

En el 2013, el Servicio de Extensión de la FNC atendió en las zonas cafeteras del departamento, de manera individual, en oficinas y fincas, a 52.790 productores y 61.563 caficultores participaron en 3.336 actividades grupales. Se continuó con el programa de certificación de fincas de Rainforest Alliance, seleccionando y adecuando fincas cafeteras del programa Nespresso, en 18 municipios de las zonas Norte y Occidente de Nariño; en el mismo año, había 1.091 fincas certificadas, lo que garantiza, a largo plazo, la economía de los productores, mejorando el ambiente y la productividad (Vanderhaegen et al. 2018). Se destaca que estos productores alcanzan un promedio de $1.451,4 \mathrm{~kg} \cdot \mathrm{ha}^{-1}$, con una inversión de \$1.638.424ha.año ${ }^{-1}$ (USD597) (FNC, 2014a).

El Grupo 2 corresponde a pequeños productores y está conformado por 36 individuos (41,86\%); un 37,14\% poseen menos de 1 ha de café. Según Pizano Salazar (2001), en Colombia predomina la pequeña explotación cafetera, que provee el 15\% de la producción total y aporta, simultáneamente, una parte significativa en mano de obra requerida por los grandes caficultores. El 74,29\% de estos productores no asisten a capacitaciones y no reciben asistencia 


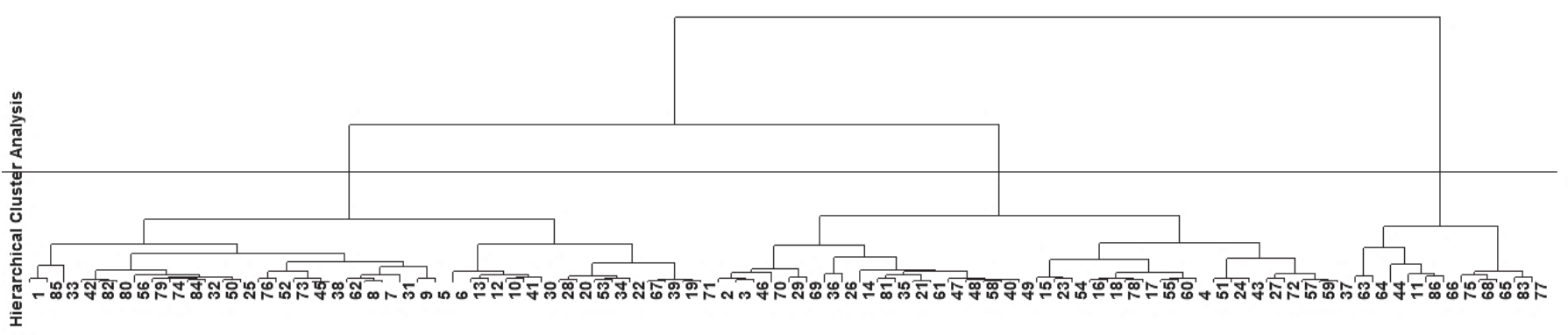

Figura 1. Conformación de grupos basados en un análisis jerárquico de las variables cuantitativas de la caracterización socioeconómica del ecotopo $220 \mathrm{~A}$.

técnica; el 80\% no tienen alianzas comerciales; el 77,14\% no pertenecen a organizaciones y no aplican BPA. Además, este grupo se caracteriza por presentar una producción de $1.169,2 \mathrm{~kg} \cdot \mathrm{ha}^{-1}$, con una inversión COP1.682.381,3ha.año ${ }^{-1}$ (USD 498,92). El Grupo 3 está conformado por 11 productores de café, que representan el $12,79 \%$. Las explotaciones cafeteras de este grupo, se caracterizaron por carecer de vivienda, ya que sus propietarios viven en el pueblo más cercano. Su promedio de producción es de $1.587 \mathrm{~kg} \cdot \mathrm{ha}^{-1}$, con una inversión de COP1.610,874ha.año ${ }^{-1}$ (USD477,72).

En general, los sistemas de producción del ecotopo $220^{\mathrm{a}}$, se caracterizan por la organización y la planificación de los mismos; las viviendas tienden a ser mejoradas en la medida en que se obtienen mejores ingresos y las familias buscan optimizar la calidad de vida, a través del trabajo y el empoderamiento de la caficultura; por ello, se interesan en capacitarse y cuentan con el apoyo de empresas, como la FNC, Fundaciones del sector y comercializadoras de café y agroquímicos. De igual modo, la mayoría de los productores tienen alianzas comerciales y pertenecen a organizaciones cafeteras; los cafetales son bien manejados, a través del conocimiento y la aplicación de las BPA, lo que conduce a que las producciones de café, en el ecotopo 220A, oscilen entre 1.204,8kg.ha ${ }^{-1}$ y $1.587 \mathrm{~kg}$. $\mathrm{ha}^{-1}$, con una inversión entre COP1.670.544ha.año ${ }^{-1}$ (USD495,41) y COP1.610.874ha.año ${ }^{-1}$ (USD477,72), sujetas a los sistemas de manejo y la capacidad de inversión de los productores.
Ecotopo 221A. El análisis de las variables cualitativas evaluadas en el ecotopo 221A mostró que el 47,9\% de los productores tienen de 1 a 3 ha de café; el 75,3\% de las viviendas son casas, de las cuales, el $57,5 \%$ tienen techo de teja; el $71,2 \%$ poseen pisos de cemento; el $63 \%$ cuentan con servicios públicos de energía y acueducto; el $74 \%$ tienen unidades sanitarias y el 57\%, pozos sépticos. El 68,5\% de las UPC registran rendimientos inferiores $1.500 \mathrm{~kg} \cdot \mathrm{ha}^{-1} \mathrm{cps}$ y el $46,6 \%$ realizan una inversión inferior a COP1.500.000 $\mathrm{ha}^{-1}$.año ${ }^{-1}$ (USD444,83). El análisis del histograma de valores propios (Tabla 3) permitió seleccionar los primeros tres factores que explican, en conjunto, el 37,17\% de la variabilidad total; el primer factor explica el $20,42 \%$ de la variabilidad, el segundo y tercer factor, el 9,53\% y $7,22 \%$, respectivamente.

En el análisis de correlación variable-factor mostró que las variables que contribuyeron en mayor proporción a la conformación del Factor 1, fueron: tipo de vivienda, techo de la vivienda, tipo de piso, combustible para preparar alimentos, servicios domésticos y servicios sanitarios, que permiten identificar al factor, como calidad de vida de los productores. Las variables que aportaron a la conformación del segundo factor o que están más correlacionadas, fueron: extensión de la finca, tipo de vivienda, tipo de piso, tipo de servicio sanitario, sistema de información de precios y aplicación de BPA. El tercer factor estuvo conformado por las variables extensión de la finca, área de café, destino de las aguas servidas y contratación de mano de obra externa (Tabla 4).

Tabla 3. Representación porcentual de la diversificación observada en las variables cualitativas relacionadas con las condiciones socioeconómicas de los productores del ecotopo cafetero $221 \mathrm{~A}$.

\begin{tabular}{|c|c|c|c|l|}
\hline Factores & Valor propio & Porcentaje & Porcentaje acumulado & \multicolumn{1}{|c|}{ Histograma } \\
\hline $\mathbf{1}$ & 0,4628 & 20,42 & 20,42 & $* * * * * * * * * * * * * * * * * * * * * * * * * *$ \\
\hline $\mathbf{2}$ & 0,2160 & 9,53 & 29,95 & $* * * * * * * * * * * * *$ \\
\hline $\mathbf{3}$ & 0,1637 & 7,22 & 37,17 & $* * * * * * * * * * * *$ \\
\hline
\end{tabular}


Tabla 4. Correlación entre las variables aportantes y cada uno de los factores principales

\begin{tabular}{|l|c|c|c|}
\hline \multirow{2}{*}{\multicolumn{1}{|c|}{ Variables }} & \multicolumn{2}{c|}{ Correlación } \\
\cline { 2 - 4 } & Factor 1 & Factor 2 & Factor 3 \\
\hline Extensión de la finca (ha) & 0,7 & 7,9 & 11,7 \\
\hline Área sembrada en café (ha) & 1 & 3,6 & 9,7 \\
\hline Tipo de vivienda & 13,5 & 13,7 & 2 \\
\hline Tipo de techo & 13,3 & 0,3 & 6,3 \\
\hline Tipo de piso & 13,5 & 14,1 & 2,2 \\
\hline Combustible para cocinar & 13,4 & 7,4 & 0,7 \\
\hline Servicios domésticos & 13,5 & 9,4 & 8,1 \\
\hline Servicio sanitario & 13,4 & 10,4 & 4,7 \\
\hline Destino final de las aguas servidas & 8,3 & 3,7 & 14,7 \\
\hline Las labores del cultivo las realiza con operarios & 0,4 & 0,8 & 13,9 \\
\hline Como se entera de los precios de venta & 1,1 & 15,4 & 7,1 \\
\hline Conoce y utiliza algunos conceptos de BPA (Buenas Prácticas Agrí́colas) & 0,1 & 10,2 & 6,1 \\
\hline Costos de insumos aplicados/año por área de café cultivado & 0,2 & 1,7 & 5,6 \\
\hline
\end{tabular}

El análisis de clasificación de fincas cafeteras del ecotopo 221A permitió la conformación de cuatro grupos bien definidos, caracterizados por su similitud intragrupal y por sus diferencias intergrupales. La conformación de los grupos y sus características, se observan en la figura 2. El Grupo 1 estuvo conformado por 41 productores, que representaron el $56,16 \%$ de la muestra total del ecotopo 221A. Este grupo, se caracterizó porque el 89,74\% cuentan con servicios de energía eléctrica y acueducto; el $97,94 \%$ de sus viviendas poseen piso en cemento y el $92,31 \%$ disponen de unidad sanitaria; se puede afirmar que este grupo corresponde a productores con mejor calidad de vida. La producción media obtenida por estos productores es de $1.225,9 \mathrm{~kg} \cdot \mathrm{ha}^{-1}$, con una inversión $\mathrm{ha}^{-1}$. año ${ }^{-1} \mathrm{de}$ COP1.751.416,05 (USD519,40).

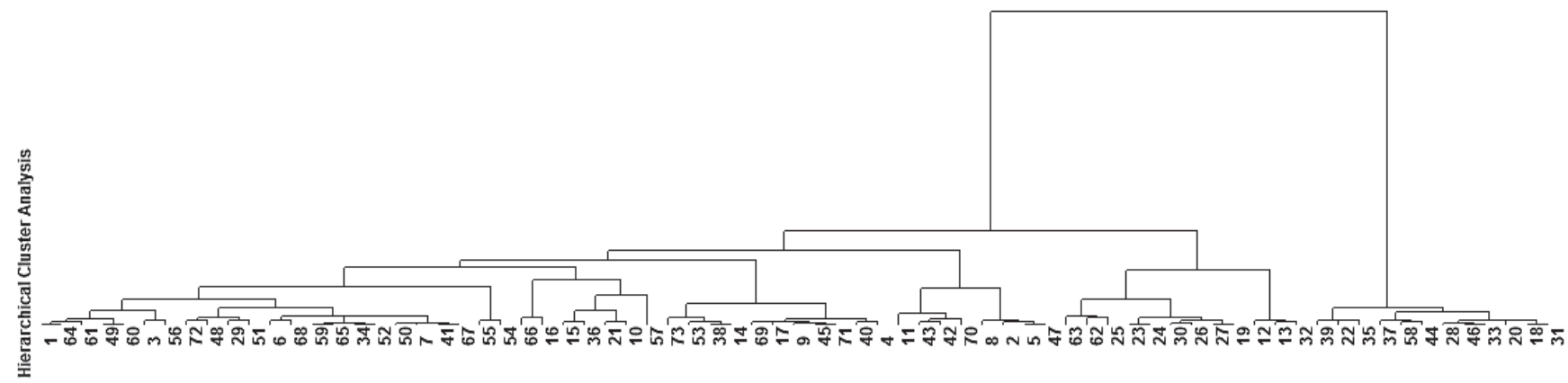

Figura 2. Conformación de grupos basados en un análisis jerárquico de las variables cuantitativas de la caracterización socioeconómica del ecotopo 221A.

El G2 estuvo conformado por ocho productores, que representan el 10,95\% de la muestra; se caracterizan porque un $75 \%$ de los productores tienen entre 3,1 y 5has de café y el 100\%, se enteran de los precios del café por radio, tv e Internet. La producción media de este grupo es de $1.150,5 \mathrm{~kg} \cdot \mathrm{ha}^{-1}$ y la inversión de $\$ 1.767 .629 \mathrm{ha}^{-1}$. año ${ }^{-1}$ (USD524,20); en el medio, se pueden catalogar como grandes productores, que han consolidado una agroempresa cafetera, con mayor inversión y rendimientos bajos. El grupo 3, lo conforman 12 caficultores, quienes representan el 16,43\% de la muestra de estudio. Los productores de esta clase, se caracterizan porque el $100 \%$ desconocen las BPA, cocinan con leña (V6=2) y se enteran de los precios del café por los vecinos; el 69,23\% de las fincas cuentan con energía eléctrica $(V 7=1)$, lo que se aproxima a los datos nacionales del 85,8\%, reportados por la FNC (2014b). La producción promedio de este grupo es de $1.002,1 \mathrm{~kg} \cdot \mathrm{ha}^{-1}$, con una inversión de $\$ 1.164 .779 \mathrm{ha}^{-1}$.año ${ }^{-1}$ (USD345,42). Estos productores son los de menor calidad de vida y los que más necesitan del apoyo estatal, para convertirse en más eficientes; los esfuerzos de las entidades encargadas de la transferencia de tecnología deben orientar sus esfuerzos hacia este grupo productivo. El cuarto grupo (G4), conformado por 12 individuos, que representan el 16,43\% de la muestra, se caracteriza porque el $92,33 \%$ de estas fincas carecen 
de vivienda. La producción promedio de este grupo es de 1.297,5 kg.ha ${ }^{-1}$, con una inversión de COP1.571.346 ha haño $^{-1}$ (USD465,99). Son productores que viven en el pueblo y manejan su finca a control remoto, reduciendo así su eficiencia productiva.

Del presente estudio, se puede concluir que los métodos multivariados aplicados permitieron determinar y comparar, de forma general, la situación socioeconómica de los cafeteros de los ecotopos 221 A y $220 \mathrm{~A}$ en el departamento de Nariño y su relación con la productividad.

En el ecotopo 220A, las variables de tipo social fueron las que más aportaron al factor 1 , mientras que en el ecotopo $221 \mathrm{~A}$, fueron aquellas variables relacionadas con la calidad de vida, las que más aportaron a la variabilidad y conformación del componente 1 .

La mayor organización y planificación de las fincas, el deseo de mejoramiento continuo mediante la capacitación y la aplicación de normas BPA, se relacionaron con mayor productividad en el ecotopo $220^{a}$. En el ecotopo 221A, se estableció menor tecnificación de los cultivos, menor receptividad a los cambios tecnológicos y menor calidad de vida, en el 16,43\% de los productores.

Conflicto de intereses. El manuscrito fue ejecutado, preparado y revisado con la participación de todos los autores, quienes declaramos que no existe conflicto de intereses que ponga en riesgo la validez de los resultados presentados. Financiación: Esta investigación fue financiada por el Sistema General de Regalías y hace parte del proyecto "Investigación y evaluación del efecto de sombra de diferentes especies arbóreas en el comportamiento agronómico y calidad de café Consacá, Nariño, occidente", liderado por el Grupo de Investigación en Producción de Frutales Andinos de la Universidad de Nariño.

\section{REFERENCIAS}

1. AGRONET. 2018. Red de información y comunicación del sector agropecuario. Disponible desde Internet en: http:/ / www.agronet.gov.co/Paginas/estadisticas.aspx (con acceso 16/04/2018).

2. ARISTIZABAL, C.; DUQUE, H. 2008. Identificación de los patrones de consumo en fincas de economía campesina de la zona cafetera central de Colombia. Cenicafe. 59(4):321342.

3. BECERRA, J. 2010. El SICA una herramienta básica para la orientación de la investigación. Chinchiná: Cenicafé. Seminario marzo 5, 2010.

4. BRAVO-MONROY, L.; POT'TS, S.; TZANOPOULOS, J. 2016. Drivers influencing farmer decisions for adopting organic or conventional coffee management practices. Food Policy. 58:49-61. https://doi.org/10.1016/j.foodpol.2015.11.003
5. BURGOS, A. 2015. Potencial del sector caficultor en Colombia, Nariño y la importancia de la responsabilidad social para su crecimiento económico. Universidad Militar Nueva Granada. Facultad de Ingeniería. Trabajo de grado para optar al título de Especialista en Gerencia Integral de Proyectos. Disponible desde Internet en: https:// repository.unimilitar.edu.co/bitstream/10654/7816/1/ BURGOSGUERRONALVAROJAVIER2015.pdf (con acceso 17/9/2018).

6. CHÁVEZ, D.; MIRANDA, I.; VARELA, M.; FERNÁNDEZ, L. 2010. Utilización del análisis de clúster con variables mixtas en la selección de genotipos de maíz (Zea mays L.). Rev. Invest. Operacional. 30(3):209- 216.

7. COMITÉ DE CAFETEROS DE NARIÑO, CCN. 2014. Labores de registro en fincas. Colombia: Sistema de información cafetero (SICA). Federación Nacional de Cafeteros.

8. CONSEJO NACIONAL DE POLITICA ECONOMICA Y SOCIAL, CONPES. 2014. Política y estrategias para el desarrollo agropecuario del Departamento de Nariño. Disponible desde Internet en: https://colaboracion.dnp. gov.co/CDT/Conpes/Econ\%C3\%B3micos/3811.pdf (con acceso 17/9/2018).

9. DE LA ROSA, M. 2011. Nariño huele a cafés especiales. Disponible desde internet en: https:/ /www.portafolio.co/ negocios/empresas/narino-huele-cafes-especiales-121486 (con acceso 17/9/2018).

10. DUSSÁN, C.; DUQUE, H.; GONZÁLES, J. 2006. Caracterización tecnológica de caficultores de economía campesina, de los principales municipios cafeteros de Colombia. Cenicafé. 57(3):167-186.

11. FEDERACIÓN NACIONAL DE CAFETEROS DE COLOMBIA, FNC. 2010. Taza de la excelencia. Disponible desde Internet en: https://www.cafedecolombia.com/ federaciondecafeteros (con acceso 17/5/2018).

12. FEDERACIÓN NACIONAL DE CAFETEROS DE COLOMBIA, FNC. 2013. Ensayos sobre Economía Cafetera. La política pública de desarrollo rural como base para la construcción de la paz. Disponible desde Internet en: https://www.federaciondecafeteros.org/static/files/ EECNo29.pdf (con acceso 10/5/2018).

13. FEDERACIÓN NACIONAL DE CAFETEROS DE COLOMBIA, FNC. 2014a. Nariño, productor de café de altísima calidad. Disponible desde Internet en: https:// www.federaciondecafeteros.org/clientes/es/sala_de_ prensa/detalle/narino_productor_de_cafe_de_altisima_ calidad/ (con acceso 10/5/2018). 
14. FEDERACIÓN NACIONAL DE CAFETEROS DE COLOMBIA, FNC. 2014b. La política cafetera 2010 2014. En: Federación Nacional de Cafeteros de Colombia. 2014. Ensayos sobre economía cafetera. Año 27 No. 30 Enero - Diciembre. Formas Finales Ltda. 128p.

15. FEDERACIÓN NACIONAL DE CAFETEROS DE COLOMBIA, FNC. 2016. Información estadística cafetera. Reportes de volumen mensual de producción, exportaciones y área cultivada. Servicios en línea FNC. Disponible desde Internet en: https://www.federaciondecafeteros. org/particulares/es/quienes_somos/119_estadisticas_ historicas/ (con acceso 18/5/2018).

16. HATANAKA, M.; BAIN, C.M.; BUSCH, L. 2005. Thirdparty certification in the global agrifood system. Food Policy 30(3):354-369. https://doi.org.10.1016/j. foodpol.2005.05.006

17. HERNÁNDEZ, L. 2009. Las denominaciones de origen como estrategia para mejorar el posicionamiento internacional de productos agroalimentarios colombianos: Caso del Café y el Banano. Facultad de Relaciones Internacionales. Universidad Colegio Mayor de Nuestra Señora del Rosario. Trabajo de grado para optar al título de Internacionalista. 57p. Disponible desde Internet en: http://repository. urosario.edu.co/bitstream/handle/10336/1491/32939151. pdf?sequence $=1$ (con acceso 10/5/2018).

18. MARTÍNEZ, F. 2004. El suelo y la producción de cafés especiales. Investigación en temas del café. Cooperativa de Caficultores del Norte. La Unión, Nariño. Colombia 10p.

19. MAURICE, N.; DAVIS, J. 2011. Unravelling the underlying causes of price volatility in world coffee and cocoa commodity markets. Disponible desde internet en: https:// ideas.repec.org/p/pra/mprapa/43813.html. (con acceso 18/4/2018).

20. MCNOUGHER, P. 2013. Insight special: Debunking coffee myths. Coffee division of ED\&F Man. Disponible desde Internet en: https://www.yumpu.com/en/document/ view/22891202/insight-special-debunking-coffee-mythsbijdendijk (con acceso 19/4/2018).

21. ORGANIZACIÓN INTERNACIONAL DEL CAFÉ, OIC. 2016. Informe de mercado del café: septiembre 2016. 5 p. Disponible desde Internet en: http://www.ico.org/ documents/cy2016-17/cmr-1216-c.pdf (con acceso 18/4/2018).

22. PANHUYSEN, S.; PIERROT, J. 2014. Barómetro de café 2014. En: Federación Nacional de Cafeteros de Colombia. Ensayos sobre economía cafetera. Año 27 No. 30 Enero - Diciembre. Formas Finales Ltda. 128p.
23. PAIVA, P. 2000. Economic and social development in Latin America: The role of Coffee. Disponible desde Internet en: http://www.ico.org/event_pdfs/paiva.pdf(con acceso 10/5/2018).

24. PIÑEROS, C. 2016. El sector cafetero colombiano como economía periférica en el sistema mundial de Wallesteirn. Universidad Militar Nueva Granada. Facultad de Relaciones Internacionales, Estrategia y Seguridad. Trabajo de grado para optar al título profesional de Relaciones Internacionales y Estudios Políticos. Disponible desde Internet en: https:/ / repository.unimilitar.edu.co/bitstream/10654/15349/3/ Pi\%C3\%B1 erosGonzal ezCamiloAndres2016.pdf (con acceso 18/9/2018).

25. PIZANO SALAZAR, D. 2001. El café en la encrucijada. Evolución y perspectivas. Bogotá: Alfaomega. 80p.

26. RÍOS, G.; ROMERO, M.; BOTERO, M.; FRANCO, G.; PÉREZ, J.; MORALES, J.; GALLEGO, J.; ECHEVERRY, D. 2004. Zonificación, caracterización y tipificación de los sistemas de producción de lulo (Solanum quitoense Lam) en el Eje Cafetero. Revista CORPOICA 5(1):22-30.

27. RODRÍGUEZ-CAMAYO, F.; LUNDY, M.; MONTENEGRO, A.; RAMÍREZ-VILLEGAS, J.; GONZÁLEZ, C.; EITZINGER, A. 2015. Planificación en zonas de conflicto y posconflicto usando evidencia científica que articuló a los sectores público y privado. CIAT Políticas en Síntesis No. 23. Centro Internacional de Agricultura Tropical (CIAT). 6p. Disponible desde Internet en: https://cgspace.cgiar.org/bitstream/ handle/10568/66317/CIAT_PB23_PLANIFICACION_ EN_ZONAS_DE_CONFLICTO_Y_POSCONFLICTO. pdf? sequence $=5 \&$ is Allowed $=\mathrm{y}($ con acceso 18/4/2018).

28. SCHEAFFER, R.L.; MENDENHALL, W.; LYMAN, O. 2007. Elementos de muestreo. $6^{a}$ edición. Ed. International Thomson, Madrid. 455p.

29. SILVA, A.; TREJOS, C. 2016. Prospectiva del café en Nariño: Sabor y aroma de una tradición. Revista Estrategia Organizacional. 5(1-2):11-28. https://doi. org/10.22490/25392786.2097

30. VANDERHAEGEN, K.; AKOYI, K.T.; DEKONINCK, W.; JOCQUÉ, R.; MUYS, B.; VERBIST, B.; MAERTENS, M. 2018. Do private coffee standards 'walk the talk' in improving socio-economic and environmental sustainability? Global Environmental Change. 51:1-9. https://doi.org/10.1016/j.gloenvcha.2018.04.014

31. VILORIA, J. 2007. Economía Regional. Banco de la Republica. Centro de Estudios Económicos Regionales (CEER) Cartagena. 88p. 